\title{
Relationship of negative and positive core beliefs about the self with dysfunctional attitudes in three aspects of life
}

This article was published in the following Dove Press journal:

Neuropsychiatric Disease and Treatment

12 October 2017

Number of times this article has been viewed

\section{Koichi Otani}

Akihito Suzuki

Yoshihiko Matsumoto

Toshinori Shirata

Department of Psychiatry, Yamagata University School of Medicine,

Yamagata, Japan
Correspondence: Yoshihiko Matsumoto Department of Psychiatry, Yamagata University School of Medicine, 2-2-2 lidanishi, Yamagata 990-9585, Japan

Tel +8I 236285322

Fax +81236285325

Email matsumotoyo@mvc.biglobe.ne.jp
Objective: Cognitive theory assumes a pivotal role of negative core beliefs about the self in dysfunctional attitudes predisposing to depression. Meanwhile, the role of positive core beliefs about the self in cognitive vulnerability to depression is unknown. Therefore, we examined the relationship of negative and positive core beliefs about the self with dysfunctional attitudes in three aspects of life.

Methods: The subjects were 311 Japanese volunteers. Core beliefs of negative-self and positive-self were evaluated by the corresponding subscales of the Brief Core Schema Scales. Dysfunctional attitudes in the areas of achievement, dependency and self-control were measured by the corresponding subscales of the 24-item Dysfunctional Attitude Scale.

Results: The negative-self subscale was correlated with the achievement, dependency and self-control subscales. The positive-self subscale was correlated with the achievement and self-control subscales.

Conclusion: The present study suggests that negative core beliefs about the self underlie all types of dysfunctional attitudes, while positive core beliefs about the self have some connections with dysfunctional attitudes related to achievement and self-control.

Keywords: negative-self, achievement, dependency, self-control, depression

\section{Introduction}

According to Beck's cognitive theory of depression, ${ }^{1,2}$ maladaptive self-schemas or dysfunctional attitudes are formed by negative experiences in childhood. They remain latent until activated by schema-congruent stressors in adulthood. These activated dysfunctional attitudes are followed by cognitive errors and automatic thoughts, which all lead to the onset and continuation of depression. Power et $\mathrm{al}^{3}$ factor analyzed 80 items of dysfunctional attitudes and obtained three factors. Based on this result, they proposed that dysfunctional attitudes are formed in three aspects of life, ie, achievement, dependency and self-control.

The cognitive theory ${ }^{1,2}$ assumes that at the deepest level of depressive self-schemas lie negative core beliefs about the self that are expressed in absolute statements such as "I am helpless" and "I am worthless". In response to these core beliefs, maladaptive self-schemas in the forms of conditions, imperatives and compensations are formed. However, this basic assumption on the role of negative core beliefs about the self in dysfunctional attitudes has never been tested directly, mainly because of lack of a reliable instrument to measure them. 
The Brief Core Schema Scales (BCSS) developed by Fowler et $\mathrm{al}^{4}$ are self-report scales to measure core beliefs about the self and others. The BCSS are composed of four subscales to evaluate four dimensions of the self and others, ie, negative-self, positive-self, negative-other and positive-other. Notably, the items of the negative-self subscale are derived from negative self-evaluations of recovered depressed patients.

In the present study, we examined the relationship of core beliefs of negative-self with the three groups of dysfunctional attitudes. Our aim was to obtain practical evidence for the involvement of negative core beliefs about the self in dysfunctional attitudes. The evaluation of core beliefs of positive-self was also included in the protocol, since it was expected that lack of these core beliefs was related to dysfunctional attitudes.

\section{Methods}

\section{Subjects}

First, 322 Japanese who declared no serious physical diseases were recruited from medical students or medical staff living in Yamagata Prefecture. Of them, nine people were revealed to have psychiatric disorders by the psychiatric screening previously described. ${ }^{5}$ These people together with two individuals with missing data were excluded, and the remaining 311 people were used for data analyses. In all, 182 were males and 129 were females. The mean (SD) age was 27.6 years ( 8.5 years). The present study was approved by the ethics committee of Yamagata University School of Medicine, and all participants gave written informed consent for the study.

\section{Assessment of core beliefs about the self and others}

Core beliefs of negative-self and positive-self were evaluated by the corresponding subscales of the Japanese version ${ }^{6}$ of the BCSS. ${ }^{4}$ This Japanese version has high reliability and validity. ${ }^{6}$ Core beliefs of negative-other and positive-other were also evaluated by the corresponding subscales of the BCSS. Each of the four subscales consists of six items. The representative items for each subscale are as follows: "I am unloved" for negative-self, "I am talented" for positive-self, "Other people are hostile" for negative-other and "Other people are accepting" for positive-other. First, participants indicate whether they hold each belief (no or yes), and if they hold the belief, then they indicate how strongly they hold it by circling one of the numbers $1-4$, in which 1 is "believe it slightly" and 4 is "believe it totally". In the participants of the present study, Cronbach's alphas for the negative-self, positive-self, negative-other and positive-other subscales were $0.83,0.85,0.76$ and 0.87 , respectively.

\section{Assessment of dysfunctional attitudes}

Dysfunctional attitudes in the areas of achievement, dependency and self-control were assessed by the corresponding subscales of the Japanese version ${ }^{7}$ of the 24-item Dysfunctional Attitude Scale (DAS-24). ${ }^{3}$ This Japanese version has been shown to have high reliability and validity. ${ }^{7}$ Each of the three subscales has eight items. The representative items for each subscale are as follows: "I must be a useful, productive, creative person or life has no purpose" for achievement, "If others dislike you, you cannot be happy" for dependency and "If I try hard enough, I should be able to excel at anything I attempt" for self-control. Participants evaluated the degree to which they agree with each statement on a 7-point scale ranging from 1 (totally disagree) to 7 (totally agree). In the participants of the present study, Cronbach's alphas for the achievement, dependency and self-control subscales were $0.79,0.61$ and 0.74 , respectively.

\section{Statistical analyses}

Participants' data were subjected to linear regression analysis and forced entry multiple regression analysis using SPSS 14.0 J for Windows (SPSS Inc., Chicago, IL, USA). In the multiple regression analysis, dependent variables were subscale scores of the DAS-24, and independent variables were those of the BCSS, age and gender. Dummy variables were used for gender $(0=$ female, $1=$ male). A $P$-value of $<0.05$ was deemed to be significant.

\section{Results}

Table 1 shows subscale scores of the BCSS and DAS-24 of the participants.

Table 2 shows correlations among all analyzed variables.

Table I Subscale scores of the BCSS and DAS-24 of the subjects BCSS

Negative-self

$4.7(4.0)$

Positive-self

$7.5(4.7)$

Negative-other

$2.2(2.7)$

Positive-other

$9.1(4.2)$

DAS-24

Achievement

$27.0(7.9)$

Dependency

$32.3(7.4)$

Self-control

$29.0(7.1)$

Note: Values in the table indicate mean (SD).

Abbreviations: BCSS, Brief Core Schema Scales; DAS-24, 24-item Dysfunctional Attitude Scale. 
Table 2 Correlations among all analyzed variables

\begin{tabular}{|c|c|c|c|c|c|c|c|c|c|}
\hline & I & 2 & 3 & 4 & 5 & 6 & 7 & 8 & 9 \\
\hline \multicolumn{10}{|l|}{ I } \\
\hline 2 & $-0.209 * *$ & & & & & & & & \\
\hline 3 & $0.301 * *$ & -0.024 & & & & & & & \\
\hline 4 & -0.069 & $0.269 * *$ & $-0.245^{* *}$ & & & & & & \\
\hline 5 & $0.350 * *$ & 0.031 & $0.219 * *$ & $-0.207 * *$ & & & & & \\
\hline 6 & $0.244 * *$ & -0.012 & 0.006 & 0.016 & $0.310 * *$ & & & & \\
\hline 7 & 0.111 & $0.167^{* *}$ & $0.131 *$ & -0.052 & $0.658^{* *}$ & $0.189 * *$ & & & \\
\hline 8 & $-0.144 *$ & -0.018 & 0.096 & 0.006 & -0.086 & -0.064 & 0.071 & & \\
\hline 9 & -0.101 & $0.116 *$ & 0.023 & -0.066 & 0.080 & $-0.152^{* *}$ & $0.116 *$ & $0.142 *$ & \\
\hline
\end{tabular}

Notes: I, negative-self; 2, positive-self; 3, negative-other; 4, positive-other; 5, achievement; 6, dependency; 7, self-control; 8, age; 9, gender. $* P<0.05$ and $* * P<0.01$.

Table 3 shows multiple regression analyses of subscale scores of the DAS-24 with those of the BCSS. The negativeself subscale was correlated with the achievement $(P<0.001)$, dependency $(P<0.001)$ and self-control $(P<0.05)$ subscales. The positive-self subscale was correlated with the achievement $(P<0.01)$ and self-control $(P<0.001)$ subscales. In the achievement subscale, the correlation with the negative-self subscale was stronger than that with the positive-self subscale, while in the self-control subscale, the opposite tendency was observed. The positive-other subscale was negatively correlated with the achievement subscale $(P<0.001)$.

\section{Discussion}

In the present study, core beliefs of negative-self were correlated with dysfunctional attitudes in the areas of achievement, dependency and self-control with varying degrees of strength. As these areas are derived from the factor analysis of all items of dysfunctional attitudes ${ }^{3}$ it is suggested that negative core beliefs about the self underlie dysfunctional attitudes as a whole. For example, a core belief of unlovability "I am unloved"4 may underlie a dependency self-schema "If others dislike you, you cannot be happy". ${ }^{3}$ Therefore, the present study reinforces the central part of cognitive theory of depression that assumes a pivotal role of negative core beliefs about the self in maladaptive self-schemas. ${ }^{1,2}$

Contrary to our expectation, core beliefs of positiveself were positively correlated with the achievement and self-control groups of dysfunctional attitudes. Notably, in the latter group, the correlation with positive-self was stronger than that with negative-self. There are two possible explanations for these apparently conflicting results. The first one, which may be particularly the case with the achievement group, is as follows. The person with this type of dysfunctional attitudes originally has core beliefs of negative-self but tries to obtain a positive self-regard by behaving according to achievement self-schemas. Until experiencing severe achievement-related stressors, this strategy is at least partly effective, resulting in the mixture of core beliefs of negativeself and those of positive-self. The second one, which may be particularly the case with the self-control group, is that the person with this type of dysfunctional attitudes has core beliefs such as "I am talented"4 and self-schemas such as "If I try hard enough, I should be able to excel at anything I attempt" ${ }^{\prime \prime}$ from the beginning. These core beliefs and

Table 3 Multiple regression analyses of subscale scores of the DAS-24 with those of the BCSS.

\begin{tabular}{llll}
\hline DAS-24 & Achievement & Dependency & Self-control \\
\hline BCSS & & & \\
$\quad$ Negative-self & $\beta=0.347^{* * *}$ & $\beta=0.265^{* * *}$ & $\beta=0.15 I^{*}$ \\
Positive-self & $\beta=0.147^{* *}$ & $\beta=0.059$ & $\beta=0.212^{* * *}$ \\
Negative-other & $\beta=0.073$ & $\beta=-0.072$ & $\beta=0.062$ \\
Positive-other & $\beta=-0.198^{* * *}$ & $\beta=-0.008$ & $\beta=-0.078$ \\
Age & $\beta=-0.052$ & $\beta=0.001$ & $\beta=0.079$ \\
Gender & $\beta=0.090$ & $\beta=-0.13 I^{*}$ & $\beta=0.089$ \\
Model summary & Adjusted $R^{2}=0.179$ & Adjusted $R^{2}=0.065$ & Adjusted $R^{2}=0.062$ \\
& $f^{2}=0.241$ & $f^{2}=0.091$ & $f^{2}=0.087$ \\
ANOVA & $F(6,304)=12.231$ & $F(6,304)=4.599$ & $F(6,304)=4.430$ \\
& $P=0.000$ & $R=0.000$ & $R=0.000$ \\
\hline
\end{tabular}

Note: $* P<0.05, * * P<0.01$ and $* * * P<0.001$.

Abbreviations: DAS-24, 24-item Dysfunctional Attitude Scale; BCSS, Brief Core Schema Scales; ANOVA, analysis of variance. 
self-schemas may be regarded as narcissistic views of the self and narcissistic expectations, respectively. If this explanation is the case, self-control self-schemas may not be that depressogenic, as we previously discussed, ${ }^{5}$ or their mechanism leading to the onset of depression may be different from that of dependency and achievement self-schemas.

As far as we know, the relationship of core beliefs about others with cognitive vulnerability to depression have never been examined. In the present study, achievement-related dysfunctional attitudes were negatively correlated with core beliefs of positive-others. This result may be related to the impersonal content of achievement self-schemas.

There are three possible limitations in the present study. First, the discussions on dysfunctional attitudes related to achievement and self-control are supported by few empirical data and, therefore, may be too hypothetical. Second, the participants in the present study were restricted in terms of age and educational level, ie, the majority were young and had received higher education. Third, they were all Japanese, who may be fundamentally different from other ethnic groups in terms of self and other evaluations. Because of these limitations, the present results are subject to replication in populations involving other age groups, educational and ethnic backgrounds.

\section{Conclusion}

The present study suggests that core beliefs of negative-self underlie all types of dysfunctional attitudes, reinforcing the central part of cognitive theory of depression. On the other hand, core beliefs of positive-self may have some connections with dysfunctional attitudes about achievement and selfcontrol, but this point should be studied further.

\section{Acknowledgments}

This study was supported by funding from the Ministry of Education, Culture, Sports, Science and Technology of Japan. This had no effect on this study.

\section{Disclosure}

All authors report no conflicts of interest in this work.

\section{References}

1. Clark DA, Beck AT, Alford BA. Scientific Foundations of Cognitive Theory and Therapy of Depression. New York: John Wiley \& Sons; 1999.

2. Dozois DJA, Beck AT. Cognitive schemas, beliefs and assumptions. In: Dobson KS, Dozois DJA, editors. Risk Factors for Depression. Oxford: Elsevier; 2008:121-143.

3. Power MJ, Katz R, McGuffin P, Duggan CF, Lam D, Beck AT. The Dysfunctional Attitude Scale (DAS). A comparison of forms A and B and proposals for a new subscaled version. J Res Pers. 1994;28(3): 263-276.

4. Fowler D, Freeman D, Smith B, et al. The Brief Core Schema Scales (BCSS): psychometric properties and associations with paranoia and grandiosity in non-clinical and psychosis samples. Psychol Med. 2006; 36(6):749-759.

5. Otani K, Suzuki A, Matasumoto Y, et al. Relationship of the 24-item Dysfunctional Attitude Scale with the temperament and character inventory in healthy subjects. Nord J Psychiatry. 2013;67(6):388-392.

6. Uchida T, Kawamura C, Mifune N, et al. The Japanese version of the Brie Core Schema Scale for schemata concerning the self and others: identification of schema patterns and relationship with depression. Jpn J Pers. 2012;20:143-154.

7. Tajima M, Akiyama T, Numa H, et al. Reliability and validity of the Japanese version of the 24-item Dysfunctional Attitude Scale. Acta Neuropsychiatr. 2007;19(6):362-367.
Neuropsychiatric Disease and Treatment

\section{Publish your work in this journal}

Neuropsychiatric Disease and Treatment is an international, peerreviewed journal of clinical therapeutics and pharmacology focusing on concise rapid reporting of clinical or pre-clinical studies on a range of neuropsychiatric and neurological disorders. This journal is indexed on PubMed Central, the 'PsycINFO' database and CAS,

\section{Dovepress}

and is the official journal of The International Neuropsychiatric Association (INA). The manuscript management system is completely online and includes a very quick and fair peer-review system, which is all easy to use. Visit http://www.dovepress.com/testimonials.php to read real quotes from published authors. 